\title{
The impact of intense fluxes of energetic protons on the low-latitude ionosphere
}

\author{
Alla Suvorova $^{1 *}$, and Alexei Dmitriev ${ }^{2}$ \\ ${ }^{1}$ SINP, Lomonosov Moscow State University, 119234 Moscow, Russia \\ ${ }^{2}$ DSSE, National Central University, 32001 Taoyaun, Taiwan
}

\begin{abstract}
Experiments on board low-Earth orbit satellites show that energetic particles (tens of $\mathrm{keV}$ ) of the Earth's radiation belt can penetrate to the equatorial ionosphere. Impact of the energetic particles on the upper atmosphere and ionosphere was studied for the case of the geomagnetic storm on 22 July 2009. We present changes of local ion concentration in the low-latitude ionosphere at night measured by the C/NOFS satellite at heights 400-800 km during the magnetic storm and quiet days. The ionospheric density during the storm was compared with a simultaneous observation of enhancements of 30-80 keV proton fluxes measured by the NOAA/POES satellites near the equator at height $\sim 850 \mathrm{~km}$. We suggest that ionospheric irregularities at night can be caused by effect of energetic protons.
\end{abstract}

\section{Introduction}

Long-term experiments on board the low-Earth orbit NOAA/POES satellites make it possible to monitor continuously the fluxes of energetic electrons and protons at altitudes $(\sim 850 \mathrm{~km})$ below the radiation belt (RB). In the region of the South Atlantic Anomaly (SAA), the RB edge dips into the topside ionosphere $(400-1000 \mathrm{~km})$ at low latitudes. Accordingly, the satellites detect consistently intensive fluxes of energetic particles there. Sometimes, at longitudes outside the SAA region, flux intensity of energetic protons or electrons suddenly and strongly increases in the low-latitude ionosphere. These particle enhancements in the ionosphere occur due to penetration of the energetic particles from the magnetosphere.

The origin of penetrating protons with energies of tens of $\mathrm{keV}$ is associated with the ring current, which develops during magnetic storms and locates at geocentric distances of several Earth radii. Energetic protons penetrate into the ionosphere at low and middle latitudes through a mechanism of charge exchange of the ring current protons on hydrogen atoms of exosphere [1].

The origin of electrons with energies of tens and hundreds of $\mathrm{keV}$ is associated with the inner RB [2, 3]. The penetration of radiation belt electrons occurs during a fast radial transport across the magnetic field lines, so-called injections $[4,5]$. The injected electrons were identified as quasi-trapped, that is their mirror points locate at altitudes significantly higher then $100-200 \mathrm{~km}$. The injected electrons penetrate at altitudes of 300 to $1000 \mathrm{~km}$

* Corresponding author: suvorova_alla@yahoo.com 
within the latitudinal region from the equator to $\pm 30^{\circ}$ of latitude. The driving mechanism of electron injections has not been established yet.

Intense fluxes of magnetospheric energetic particles penetrating to low altitudes affect the upper atmosphere and the ionosphere. Particularly, the particles ionize and excite neutral species altering local characteristics of the ionosphere. The ionization effect produced by energetic electrons in the low-latitude ionosphere was studied comprehensively [2-4]. It was found that long-lasting great enhancements of energetic electron fluxes effectively ionize the thermosphere at heights of 300-500 km that result in significant increase of the total electron content (TEC) in the F-region of the ionosphere. To our knowledge, studies of impact of proton enhancements on the low-latitude ionosphere have not been reported. We present here the results of the analysis of simultaneous local ionospheric ion density perturbations and energetic proton flux enhancements during the geomagnetic storm on 22 July 2009.

\section{Data}

In situ measurements of ion concentration and fluxes of energetic protons were made on board the low-Earth orbit satellites. The database of the measurements are available on the CDAWEB cite [6]. Data on protons with energies 30-80 keV in the period 21-22 July 2009 were gathered from two detectors installed on each of the five NOAA/POES satellites. The satellites have circular polar Sun-synchronous orbits at altitudes of $\sim 850 \mathrm{~km}$ and with period of $100 \mathrm{~min}$. In average, the orbital planes were located at the following local times within \pm 1 hr: 6-18, 9-21, and 2-14 LT. Two identical detectors measures proton fluxes in two directions: along and perpendicular to the local vertical direction [7]. In the study, we use both detectors to calculate the aggregate flux of quasi-trapped and precipitating protons with time resolution of $16 \mathrm{~s}$.

Data on ion concentration with time resolution of $0.5 \mathrm{~s}$ was acquired from the CINDI instrument installed on board the C/NOFS satellite. The satellite experiment was developed for investigation of scintillations in the Earth's ionosphere. The satellite had elliptical orbit with period of $97 \mathrm{~min}$ and inclination of $13^{\circ}$, a perigee of $400 \mathrm{~km}$ and an apogee of $850 \mathrm{~km}$. On 21 -22 July 2009, the satellite passed the dayside being near the orbital perigee and the nightside - near the apogee.

\section{Results}

Fig.1 shows geomagnetic activity indices SYM-H and Kp on 21-22 July 2009 [8, 9]. The magnetic storm on 22 July was moderate with maximal indices $\mathrm{Kp} \sim 6$ and SYM-H $\sim-90 \mathrm{nT}$. The previous day of 21 July was magnetically quiet. The main phase of the magnetic storm began on 22 July 2009 after 0 UT and the recovery phase started after 8 UT. The SYM-H index exhibits an intensification of the ring current during the main phase from 0 to $6 \mathrm{UT}$. Then after a partial recovery, another short intensification has occurred. We compare the data on the ionospheric plasma density and energetic protons observed during the main phase of the storm with the data during the magnetic quiet condition.

\section{1 lonospheric activity during the storm}

Fig.2 (a,b) shows ion concentrations in the ionosphere measured along the C/NOFS orbit from 4 to 9 UT on July 22 and 21, respectively, during the storm and the quiet day. The time interval includes three consecutive revolutions $\mathrm{A}, \mathrm{B}$, and $\mathrm{C}$ on each day. Fig.3 (a,b) shows the orbits $\mathrm{A}, \mathrm{B}, \mathrm{C}$ in geographic coordinates. It should be noted that physical parameters 
measured on the corresponding orbits during two consecutive days, can be compared, despite a small shift of the orbits in longitude (about $15^{\circ}$ ).

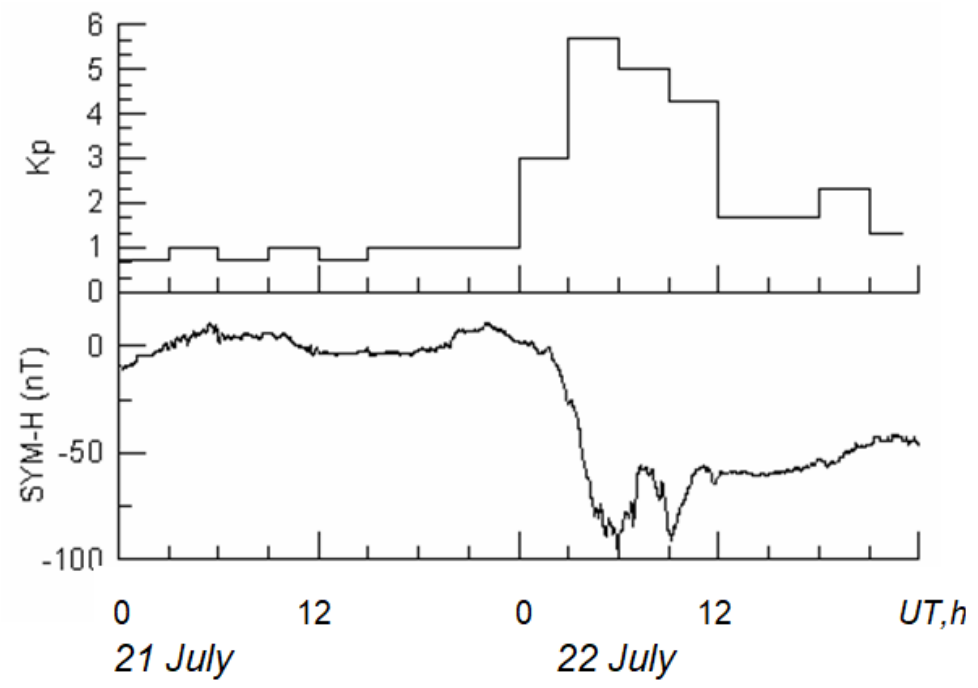

Fig. 1. Geomagnetic activity on 21-22 July 2009: (top) Kp index and (bottom) SYM-H index. A moderate magnetic storm started at 0 UT on 22 July.

(a) Storm

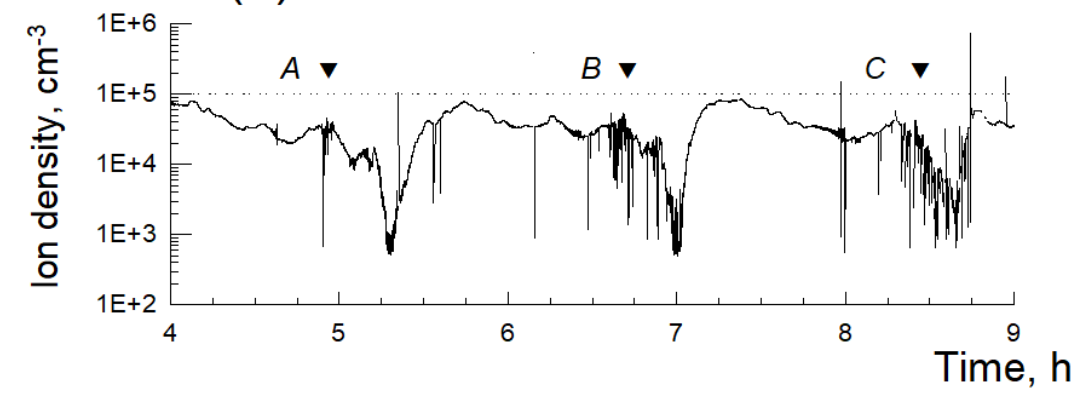

(b) Quiet

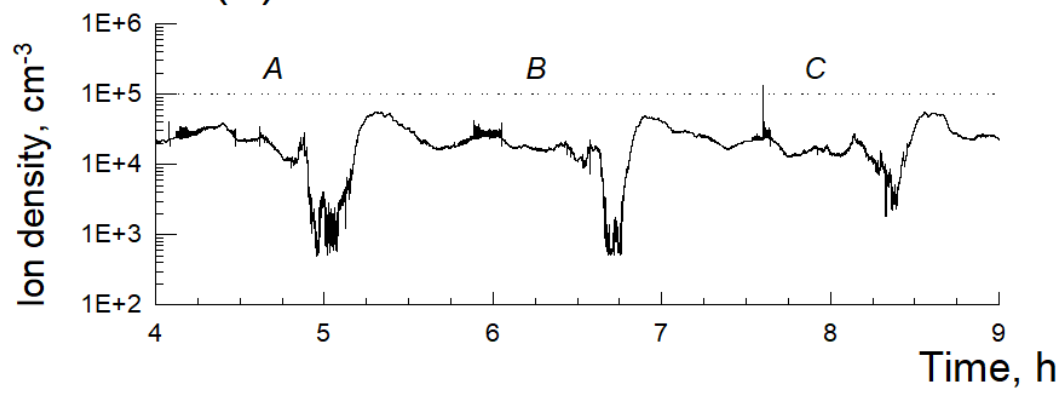

Fig. 2. Time profiles of ion concentration obtained at the C/NOFS satellite from 4 to 9 UT during (a) the magnetic storm on 22 July and (b) magnetic quiet on 21 July 2009. The satellite revolutions are marked by A, B, C. The ionospheric irregularity structures indicated by symbol $\boldsymbol{\nabla}$ were observed along the orbit at night time during the storm. 
One can see that during the quiet day, maximal ion concentration of $\sim 5 \cdot 10^{4} \mathrm{~cm}^{-3}$ was observed at $\sim 5: 15, \sim 6: 55$ and $\sim 8: 30$ UT (Fig.2b). At that time, the satellite was in the southern-eastern sector (the orbital parts A, B, C colored by red in Fig.3b). For example, at 6:55 UT the satellite was located at longitude of $7^{\circ}$ moving along the ascending part of the orbit B in the morning sector at $\sim 7$ LT (Fig.3b). At that time, the altitude was increasing from the perigee to $450 \mathrm{~km}$. Therefore, three smooth peaks of the density in the time profile (Fig.2b) were detected within the dayside crest of the equatorial ionization anomaly (EIA) and slightly above the F-layer maximum.
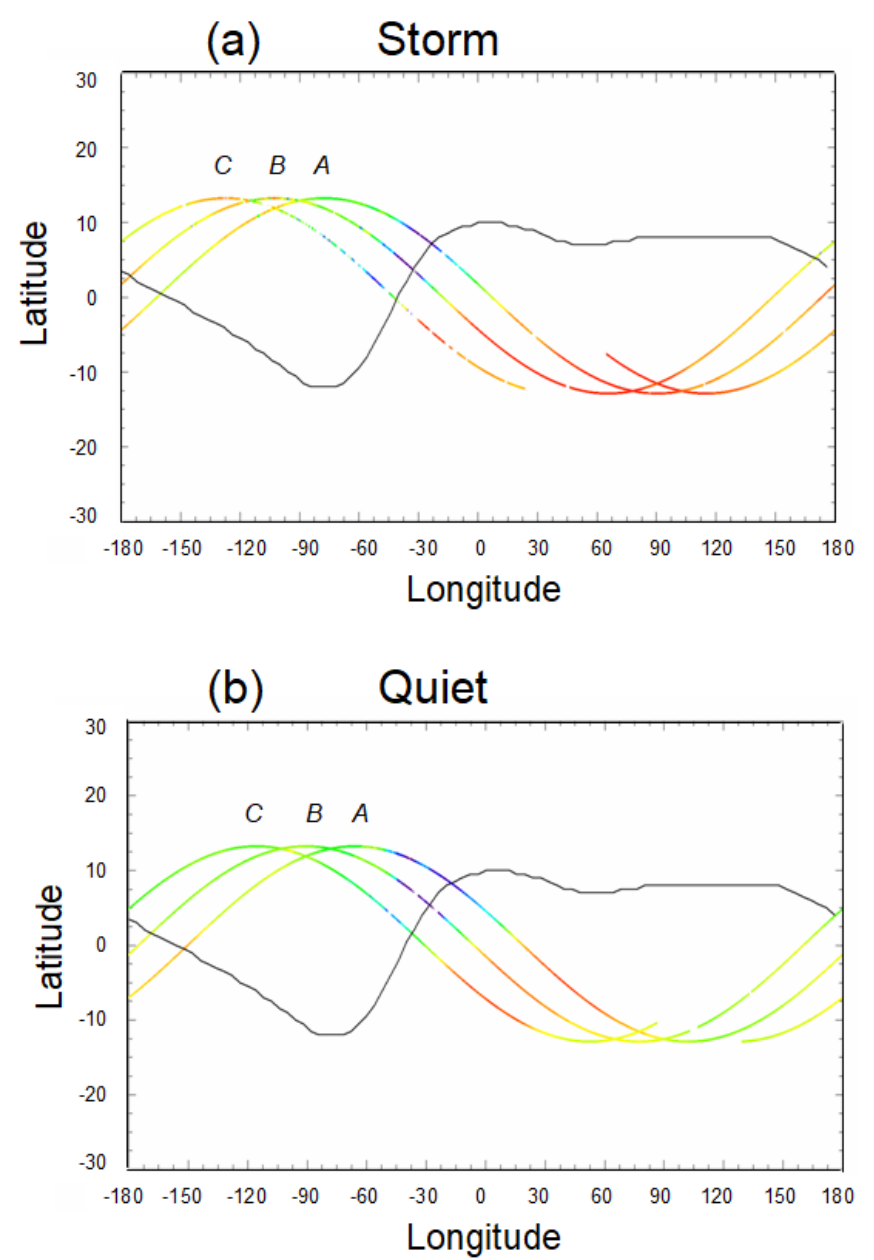

Fig. 3. Geographic coordinates for three consequent revolutions (A,B,C) of the C/NOFS satellite from 4 to 9 UT on (a) 22 July 2009 and (b) 21 July 2009. The value of ion concentration along the orbit is indicated by colors: red corresponds to the maximum value and violet corresponds to the minimum value. The black curve shows the magnetic dip equator.

In Fig. $2 \mathrm{~b}$, prior the density peaks, deep decreases with minimal ion concentration of $<10^{3}$ $\mathrm{cm}^{-3}$ were observed at $\sim 5$ UT and $\sim 6: 45$ UT. At that time, the satellite moved along the descending part of the orbits in the dawn sector ( 4 LT) at altitudes decreasing from $450 \mathrm{~km}$ to the perigee (see the orbital parts A, B, C colored by violet in Fig.3b). In Fig.3b, the minimum density is detected around the magnetic dip equator and, hence, it is the EIA trough. Note that the ion density in the EIA trough is less then that in the night sector and at higher 
altitudes. For example, in the interval 6:15 - 6:30 UT, the satellite detected the ion density of $\sim 2 \cdot 10^{4} \mathrm{~cm}^{-3}$ at night (21-02 LT), when it was moving in the topside ionosphere at altitudes above $500 \mathrm{~km}$, but this value was higher then the value in the trough.

Ionospheric ionization at low latitudes increases in response to magnetic storms, known as an ionospheric positive phase [10]. In Fig.2a, the ion concentrations along the orbits increased by 1.5-2 times from morning to night during the storm main phase in comparison with the quiet conditions. We should pay attention that in the topside ionosphere at night time ( 22-03 LT) the ion concentration highly fluctuated. Regions of the night ionospheric irregularities were detected in the northern-western sector at altitudes between 500 and 800 $\mathrm{km}$. The focus of our study is this ionospheric activity feature arose during the storm main phase. In the next section we exam a possible effect of energetic particles on the nighttime ionosphere.

\subsection{Precipitations of the ring current protons during the storm}

Fig. 4 shows geographic distribution of fluxes of $30-80 \mathrm{keV}$ protons detected in the time interval 4 - 9 UT during the storm on July 22. For comparison, Fig.5 shows proton fluxes during the quiet day on July 21 . One can see that proton fluxes at low latitudes increased significantly during the storm main phase. Particularly, we pay attention on enhancements in the western hemisphere at longitudes from $-100^{\circ}$ to $-150^{\circ}$, i.e., in the same region where the ionospheric irregularities were observed.

Apparently, the energetic protons are originated from the storm-time ring current. Due to the charge exchange mechanism, the protons penetrate to low latitudes and impact the ionosphere from the top to the bottom. The impact is non uniform in latitudes and heights that might produce local inhomogeneities in the ionospheric ionization.

\section{Summary}

According to the recent researches of the influence of the magnetosphere to the ionosphere, intense fluxes of energetic particles can penetrate to the ionosphere at low latitudes and play an important role in the processes associated with additional ionization and plasma inhomogeneity in the F region. In this work, we conduct a comparative analysis of the data on ion concentration and fluxes of the $30-80 \mathrm{keV}$ protons measured in situ at the same time and in the same region of the low-latitude ionosphere during the magnetic storm on 22 July 2009. During the storm main phase, we observed intensive fluxes of the protons penetrating from the storm ring current to low latitudes and height of $850 \mathrm{~km}$ in the night sector. At that time, variations of the ion concentration at heights $500-700 \mathrm{~km}$ are featured by largeamplitude fluctuations. It can be suggested that formation of the night time ionospheric irregularities at low latitudes is caused by the impact of energetic protons on the topside ionosphere. A mechanism of this phenomenon is a subject of future studies.

We acknowledge a team of the NOAA/POES satellites for providing experimental data on energetic particles and CDAWEB for providing experimental data on ionospheric concentration from the $\mathrm{C} / \mathrm{NOFS}$ satellite. The SYM-H and Kp indices was provided by the WDC for Geomagnetism, Kyoto (http://wdc.kugi.kyoto-u.ac.jp/wde/Sec3.html). The work of A.V.D. was supported by grant MOST 108-2111-M-008-035 to National Central University. 


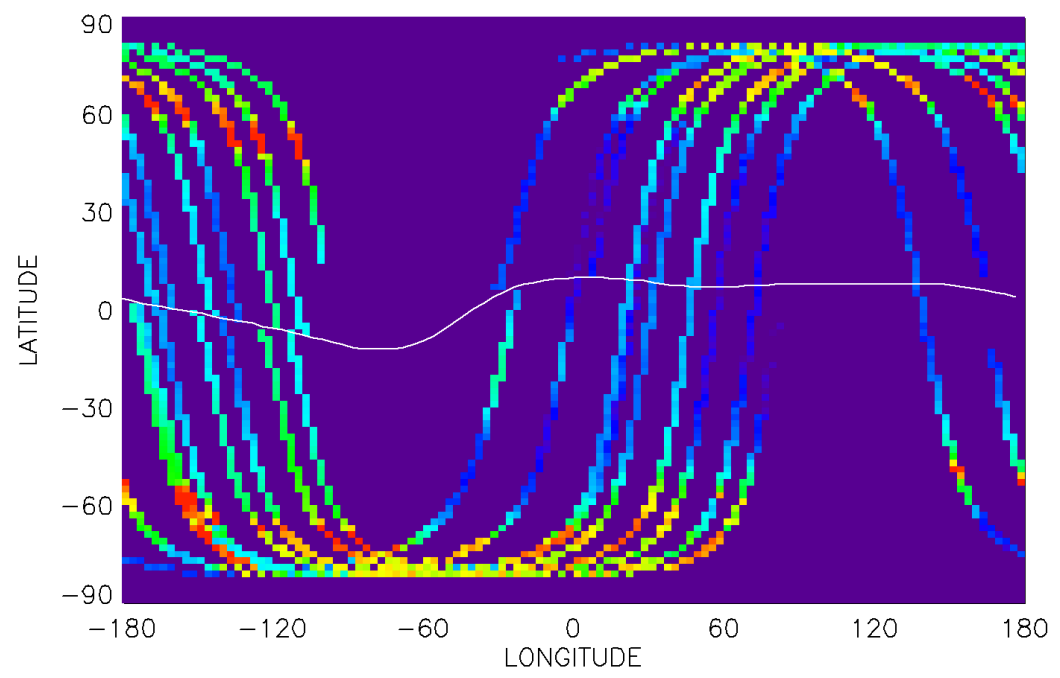

Fig. 4. Fluxes of $30-80 \mathrm{keV}$ protons in geographic coordinates from 4 to 9 UT during the storm on 22 July 2009. White curve shows the magnetic dip equator.

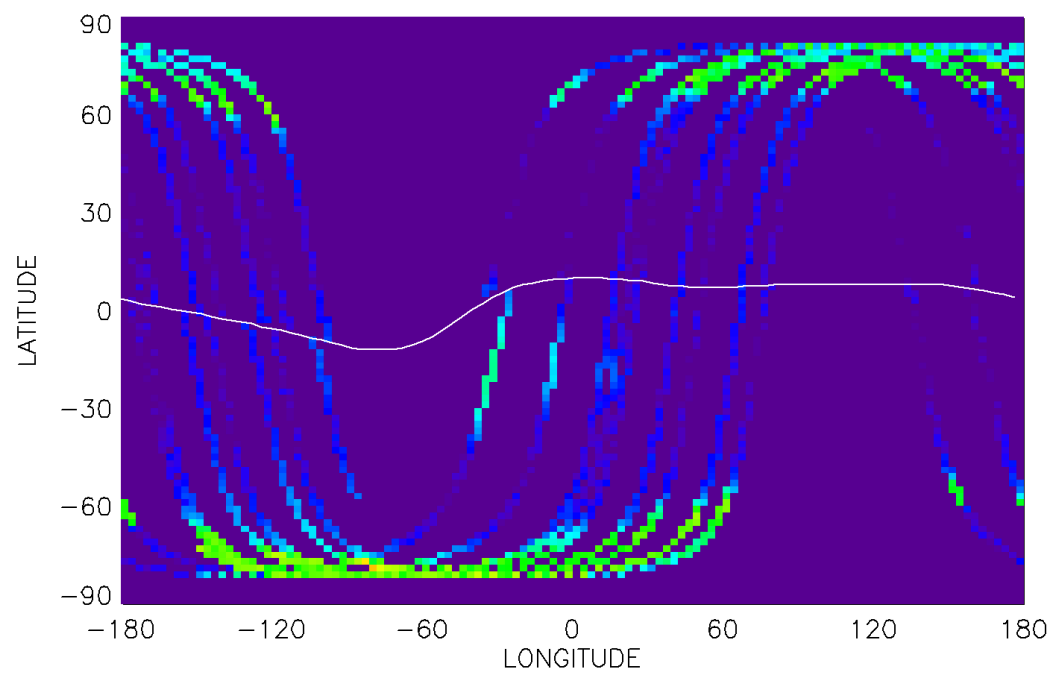

Fig. 5. Fluxes of $30-80 \mathrm{keV}$ protons in geographic coordinates from 4 to 9 UT during the quiet day on 21 July 2009. White curve shows the magnetic dip equator.

\section{References}

1. L.R. Lyons, D.J. Williams, Quantitative Aspects of Magnetospheric Physics (D. Reidel Pub. Co., Dordrecht Boston, 1984)

2. A.V. Suvorova, L.-C. Tsai, A.V. Dmitriev, Planet. Space Sci., 60, 363-369 (2012)

3. A.V. Suvorova, C.-M. Huang, H. Matsumoto, A.V. Dmitriev, V.E. Kunitsyn, E.S. Andreeva, I.A. Nesterov, L.-C. Tsai, J. Geophys. Res. Space Physics, 119, 9283-9303 (2014) 
4. A.V. Suvorova, A.V. Dmitriev, L.-C. Tsai, V.E. Kunitsyn, E.S. Andreeva, I.A. Nesterov, L.L. Lazutin, J. Geophys. Res., 118, 4672-4695 (2013)

5. A.V. Suvorova, A.V. Dmitriev, Radiation aspects of geomagnetic storm impact below the radiation belt. Book Chapter in Cyclonic and Geomagnetic Storms: Predicting Factors, Formation and Environmental Impacts, Ed. Victoria P. Banks (NOVA Science Publishers, Inc., New York, 2015)

6. URL: https://cdaweb.gsfc.nasa.gov/index.html

7. D.S. Evans, M.S. Greer, Polar orbiting environmental satellite space environment Monitor: 2. Instrument descriptions and archive data documentation. (Tech. Memo V. 1.4. Colorado: NOAA Space Environment Laboratory, 2004)

8. URL: http://wdc.kugi.kyoto-u.ac.jp/kp/index.html\#LIST

9. URL: http://wdc.kugi.kyoto-u.ac.jp/aeasy/index.html

10. M. Mendillo, Rev. Geophys., 44, RG4001 (2006) 